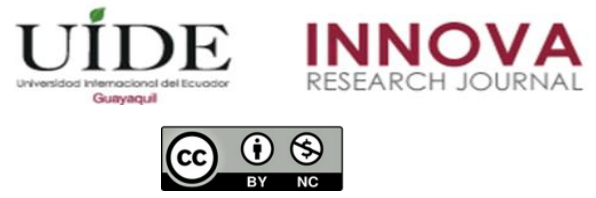

INNOVA Research Journal, ISSN 2477-9024

(Enero - Abril 2021). Vol. 6, No.1 pp. 61-83

DOI: https://doi.org/10.33890/innova.v6.n1.2021.1566

URL: http://revistas.uide.edu.ec/index.php/innova/index

Correo: innova@uide.edu.ec

\title{
El desempeño laboral e intención de rotación de los trabajadores de la Unidad de Recursos Humanos de la DRELM, un análisis cualitativo
}

\section{Work performance and intention to rotate workers in the DRELM Human Resources Unit, a qualitative analysis}

\author{
Rogelio Espinoza Galvez \\ (D) https://orcid.org/0000-0003-3669-7885 \\ Universidad César Vallejo, Perú \\ Olga María Del Carmen Ramos Huamán \\ (iD) https://orcid.org/0000-0002-7872-3597 \\ Universidad Norbert Wiener, Perú \\ Miryam Aracelli Espinoza Gonzáles \\ (iD) https://orcid.org/0000-0003-3508-8463 \\ Universidad César Vallejo, Perú \\ Pompeyo Gabriel Asca Agama \\ (D) https://orcid.org/0000-0001-8819-3318 \\ Universidad César Vallejo, Perú
}

Autor para correspondencia: respinoza22@ucvvirtual.edu.pe; olga.ramos@uwiener.edu.pe; mespinozagon@ucvvirtual.edu.pe; pascaa@ucvvirtual.edu.pe

Fecha de recepción: 22 de septiembre de 2020 - Fecha de aceptación: 02 de octubre de 2020

\section{Resumen}

El objetivo general de esta investigación fue analizar el desempeño laboral y la intención de rotación de los empleados de la unidad de talento humano de la DRELM, durante el año 2018. El enfoque utilizado fue el cualitativo, con estudio de casos, trabajo inductivo, con revisión documental y entrevistas estructuradas como método para recolección de información. Se transcribieron las entrevistas para triangularlas posteriormente en una matriz para ese efecto y así poder realizar un análisis comparativo con lo que se obtuvo resultados que se espera permita a la institución objeto del estudio definir mejoras en los procesos establecidos para administrar al recurso humano y obtener mejores niveles de desempeño. Se trabajó con tres grupos de trabajadores reconocidos por el número del decreto con el que fueron contratados; los resultados mostraron que el desempeño laboral de los distintos grupos fue deseable, óptimo en dos grupos aun cuando existen diferencias de remuneraciones o no tener indicadores con los que sean medidos. Con el tercer grupo el desempeño no es determinante ni óptimo debido a la falta de especialización del grupo. Con respecto a la intención de rotación se encontró que existe un claro deseo de rotación en los trabajadores. 
Palabras claves: Desempeño laboral; Intensión de rotación; Régimen laboral.

\begin{abstract}
The general objective of this research was to analyze the job performance and the intention to rotate the employees of the DRELM human talent unit, during 2018. The approach used was qualitative, with case studies, inductive work, with documentary review and structured interviews as a method for gathering information. The interviews were transcribed to triangulate them later in a matrix for that purpose and thus be able to carry out a comparative analysis with which results were obtained that are expected to allow the institution under study to define improvements in the processes established to manage human resources and obtain better performance levels. We worked with three groups of workers recognized by the number of the decree with which they were hired; the results showed that the work performance of the different groups was desirable, optimal in two groups even when there are differences in remuneration or when there are no indicators with which they are measured. With the third group, performance is neither decisive nor optimal due to the group's lack of specialization. Regarding the intention to rotate, it was found that there is a clear desire for employee rotation.
\end{abstract}

Keywords: Job performance; Intension of rotation; Job regime.

\title{
Introducción
}

En la década de los 80s la OCDE Organización para la Cooperación y el Desarrollo Económico reportó que los niveles de desempleos europeos eran mayores que los presentes en USA y Canadá (OCDE; 1990), se encontraron tres causas posibles: (1) la tecnología y su aceleración evolutiva; (2) una competencia más agresiva por parte de los países con mayor desarrollo y (3) el aumento del precio del petróleo. Chávez (2001) encontró que la excesiva rigidez de las políticas laborales sería la razón por la que la tasa de desempleo se elevaba. Para los 90s se encontró una definición nueva en el contexto laboral que se contraponía a la rigidez, esta era sustentada en el principio constitucional de estabilidad laboral: la flexibilización del trabajo, lo que representaba cambios en el trabajo determinados por la necesidad de empleados especializado, con capacidad para ocupar cargos en diferentes niveles. (Tostes y Villavicencio 2012)

CEPAL (2012) resaltó el antecedente que sirvió de base para reformular el sistema laboral peruano; las normas anteriores eran un obstáculo para que la población mayoritariamente no cuente con un trabajo formal, sumado a la escasa profesionalización de los funcionarios y servidores públicos, la permanente rotación en el personal y las dificultades generalizadas en la carrera administrativa pública. El Perú, en cuanto a derechos y obligaciones de los empleados estatales en los últimos 25 años ha sufrido cambios de forma acelerada pero sin planificación, dentro del marco de la nueva gestión pública, el crecimiento del número de empleados estatales, desembocando en la creación de regímenes laborales que más allá de solucionar los inconvenientes en la gestión y administración del personal forjo ingobernabilidad, caos, desorden, injusticias, discriminación entre trabajadores en una misma institución y la intromisión política en el acceso a los cargos.

Esta obra se comparte bajo la licencia Creative Common Atribución-No Comercial 4.0 International (CC BY-NC 4.0) Revista de la Universidad Internacional del Ecuador. URL: https://www.uide.edu.ec/ 
La convivencia de distintos regímenes laborales, con diferencias remunerativas, mejor retribución en uno, óptimas condiciones laborales en otro, fue forjando descontento, desorientación o la intención de rotación interna del personal; en suma generaron conflictos en la organización entre trabajadores en un mismo régimen, crecimiento exponencial del número de servidores administrativos con reglas y condiciones diferentes, un régimen con compensaciones inequitativas, discriminatorias; con un desempeño laboral desmotivado, conformista y en algunos casos de rebeldía y eventualmente propensos a dejarla. La Unidad de Recursos Humanos (URH) de la DRELM implementó la política de personal definido por las políticas de la Dirección, que en muchos casos dejo de lado el régimen general bajo su administración, no se implementaron acciones para evaluar el desempeño laboral, el rendimiento individual o colectivo, la identificación institucional, las necesidades de los servidores y su intención de rotación.

En estudios previos, diferentes autores que analizaron las mismas variables en otros países coinciden en que existen preocupaciones para conservar e incentivar las relaciones interpersonales que permitan mantener buenos ambientes laborales y al mismo tiempo mantener procedimientos que garanticen higiene y seguridad en el trabajo. De la misma forma se menciona la importancia implementar instrumentos de evaluación del desempeño laboral y permitir que se generen verdaderas oportunidades de crecimiento dentro de las instituciones públicas, lo que permitiría reducir los niveles de rotación y mejora del desempeño (Buitrago, Constan y Lemus 2016; Chiang y San Martín, 2015; Gruezo, 2017; Matzmura, 2018: Villegas, 2012).

En el caso peruano, autores han concluido que los tipos de contratos presentes en el sector y el desempeño son variables dependientes; existiría una correspondencia inmediata y significativa entre los diferentes regímenes laborales presentes y los resultados obtenidos, debido a que hay grandes diferencias entre las condiciones laborales de los diferentes grupos, tal como se expuso anteriormente, esto genera mayores niveles de insatisfacción ya que los distintos grupos coexisten generando distintas clases de empleados en las mismas instituciones públicas (Castellanos, 2017; Cepeda, 2017; Mariscal y Arias, 2018; Rueda, 2011; Ruiz, 2017). Se concluyó que los recursos humanos del Estado sujetos a contratos por SNP - terceros no cuentan con derechos y beneficios laborales lo que significa una evidente trasgresión de la norma laboral, a pesar de las leyes que reivindican sus derechos ante una acción de amparo. El contrato CAS es de condición laboral, como se reconoció en la más alta instancia Constitucional donde reconoce derechos y beneficios laborales. Aun así, Maldonado, Monteza y Rosales (2018) encontraron que los trabajadores con mayor antigüedad, tienen mayor intención de dejar la organización, y mayores probabilidades de presentar conductas y comportamientos organizacionales negativos.

A partir de la revisión del estado del arte se definen las categorías siguientes: desempeño laboral, que es el proceso que se dirige hacia la obtención de los objetivos por parte de los colaboradores que permiten a su vez conseguir las metas grupales y la gestión del desempeño es una secuencia dinámica y permanente delimitada por acciones y procedimientos que pueden significar una fortaleza para la organización de acuerdo con los resultados obtenidos (Chiavenato, 2014; Wayne, 2010).

De acuerdo con Maslow (1943) las personas tienen necesidades ordenadas en una jerarquía, en la medida que van satisfaciendo necesidades básicas, los sujetos van desarrollado nuevas necesidades menos básicas; Herzberg et al. (1959) complementó esta idea con la Teoría

Esta obra se comparte bajo la licencia Creative Common Atribución-No Comercial 4.0 International (CC BY-NC 4.0) Revista de la Universidad Internacional del Ecuador. URL: https://www.uide.edu.ec/ 
de dos factores: satisfactores e insatisfactores que permiten que las personas alcancen sus metas con mayor o menor voluntad. Por otra parte, McGregor (1960) propuso la Teoría X y Teoría Y: antepuestas en sí, la primera es creencia de los gerentes que los empleados trabajan bajo amenazas, la segunda teoría los jefes opinan que los servidores aspiran y necesitan trabajar.

Campbell et al. (1970) definió el desempeño como una conducta laboral que involucra la obtención de los fines institucionales, ellas comprenden algún tipo de beneficio. En ese sentido, toda institución estatal o privada asume claramente sus objetivos, sus metas, la misión y visión a alcanzar en un determinado plazo o lapso de tiempo; ello es poco probable o casi nunca logrado cuando dentro de la institución estas no son dadas a conocer a sus trabajadores. Asimismo, Drucker (2002) manifestó su inquietud sobre el desempeño laboral, planteando que debe construirse nuevos enunciados, señalando reformadas o aproximaciones en formato no financiero. Sonnentag y Frese (2012) puntualizaron que las instituciones necesitan colaboradores con un óptimo desempeño para lograr las metas, entregar productos que les permita tener una ventaja competitiva. Robbins y Coulter (2010) el desempeño concurre en la conclusión de la acción, aun cuando ella, simbolice horas intensas previas de entrenamiento, así como desarrolla responsabilidades funcionales eficientes y eficazmente posibles. Chiavenato (2014) refirió que el desempeño es la eficacia del trabajador, siendo necesaria para ella, que el individuo desarrolle sus funciones, realizando una gran labor y que con ello obtenga la satisfacción laboral.

Wayne (2010) planteó como sub categorías: (1) competencia donde incluyó conocimientos, destrezas, atributos del temperamento, modos de conducta que pueden ser de naturaleza técnica, relacionadas con destrezas interpersonales; (2) logro de las metas, que significa que los fines son más transcendentales que los medios, como un factor a evaluar considera los resultados en el logro de las metas; el individuo o el grupo debe controlar esos resultados que deben conducir al éxito; (3) potencial de mejoramiento, que se refiere a la valoración del desempeño de los empleados basados en históricos mientras que deberían esperar mejoramiento en comportamientos y resultados futuros para la obtención de metas personales y organizacionales

Se define la intención de Rotación, como la posibilidad que los empleados abandonen la organización; la rotación es el abandono de los trabajadores y estos son reemplazados por otros, cuando el reemplazo no se da, se produce la disminución de personal (Testa, 1973). Se han propuesto tres maneras de tránsito laboral: (a) la rotación voluntaria, cuando el empleado por razones personales o profesionales termina su relación laboral con la empresa; (b) la rotación voluntaria inevitable decisión vital del empleado, sin control del empresario y (c) la rotación involuntaria cuando la dirección decide no renovar o terminar una relación laboral dada una necesidad económica o una mala relación entre el empleado y la institución (Rodríguez, 1998; Taylor, 1999).

Otros autores han señalado que la rotación puede ser afectada por distintos factores, entre ellos la necesidad de las personas de suplir necesidades que podrían no ser satisfechas en un mismo lugar de trabajo por un período prolongado. Los cambios propios de los negocios, las etapas del ciclo de vida del mismo también pueden generar mayor o menor rotación, disminución de la productividad, cambios medioambientales que generan que se deba buscar una selección óptima de los trabajadores junto con mejores remuneraciones con el fin de mejorar el clima 
empresarial; así mismo se estableció la causalidad entre las compromiso organizacional con la intención en la organización, el compromiso afectivo junto con la satisfacción laboral, así como las necesidades propias personales afectarán la intención de permanecer en la empresa (Bohlander y Snell, 2013; Chiavenato, 2011; Mobley, 1977; Mowday, Porter y Steer, 1982; Porter y Steer, 1973; Robbins y Judge, 2009; Vázquez, 2001; Zimmerman et al, 2006).

De acuerdo con Littlewood (2006) se establecen las subcategorías de la intención de la rotación: (1) apoyo organizacional percibido, que es la percepción de los empleados sobre el interés de la empresa en su personal, otros autores encontraron que el apoyo organizacional es comparado con la teoría del intercambio social (Eisenberger, et al, 1986 ;Wayne et al., 2010); (2) Compromiso organizacional, una de las variables más estudiadas debido a su importancia en los resultados en las organizaciones, de acuerdo con Meyer y Allen (1991) el compromiso organizacional tendría dimensiones, el compromiso normativo y el afectivo, este es uno de los modelos más aceptados y utilizados en los distintos estudios.

La subcategoría (3) es la búsqueda de empleo, es la averiguación consciente y activa de un nuevo empleo en otra organización, con la finalidad de comparar de forma activa ante otras posibilidades y cambiar de trabajo (Littlewood, 2006; Mobley (1977) incorporo una propuesta de rotación de personal en la búsqueda de empleo, como una condición en la intención de continuar, donde los empleados insatisfechos son más perceptivos a ofertas laborales y al cambio de empleo. Para Griffeth, Hom y Gaertner (2000) es la indagación de empleo y como buscarlos es la razón propia de la varianza del propósito de permanencia, consecuentemente, buscar empleo es el inicio de la intención de permanencia o viceversa.

La subcategoría (4) intención de permanencia, es definida como el esquema de la finalidad de permanencia, intención forjada y consciente de continuar en la empresa (Fishbein y Ajzen, 1975; Hom y Griffeth, 1991; Littlewood, 2006; Tett y Meyer, 1993; Vandenberg y Scarpello, 1990); la sub Categoría (5) Rotación voluntaria de personal, se definió como la salida que un empleado prepara y ejecuta por cuenta propia con la intención de terminar la relación laboral, por medio de la renuncia o el abandono, en ese sentido, se observa empleados que se separan y empleados que permanecen por un lapso en la institución (Littlewood, 2006; Sánchez, 2017).

Al estudiar en esta investigación empresas públicas es importante definir el concepto de Régimen laboral, que tiene su génesis en la Constitución Política de 1979 y en la de 1993 (Sánchez, 2003); Rodríguez (2008) precisó que la estructura normativa constitucional, admitió la coexistencia de regímenes generales: el público y el privado. Espinal (2008) define el régimen laboral como el contexto jurídico en que se encuentra inmersos la relación laboral de persona natural con otra, pudiendo esta ser natural o jurídica, aplicándosele un cumulo de normas legales que regulan derechos y beneficios.

Castillo (2011) refirió que se segmenta en nuestra carta magna dos regímenes bien definidos, público y privado, dentro del primer grupo se encuentra los trabajadores de una organización del estado, normado en el D. L. N ${ }^{\circ} 276$, el D. L. $\mathrm{N}^{\circ} 1057$ y los servicios no personales del D. L. N $\mathrm{N}^{\circ}$ 30225; en el segundo grupo está el sistema privado regulado por el D. L. No 728 "Ley de productividad y competitividad laboral". 
De acuerdo con los documentos revisados y las norma constitucional del régimen público, para efectos de este estudio se propuso como subcategorías los siguientes regímenes de los que se escogieron los participantes: (1) Decreto Legislativo $\mathrm{N}^{\mathrm{o}} 276$, en esta norma se daba mayor espacio a trabajadores de carrera administrativa y estos eran clasificados en 14 niveles según el artículo $10^{\circ}$, al Grupo Profesional concierne 08 niveles superiores; el Grupo Técnico 10 niveles, entendidos del 3ro., al 12vo.; y los 07 niveles inferiores al Grupo Auxiliar (Cabrera y Salazar, 2005); (2) Régimen de la Contratación Administrativa de Servicios - CAS, D. L. No 1057, reglamentada con el D. S. N 075-2008, se creó con la intención de viabilizar los Acuerdos del TLC con Estados Unidos y obteniendo su reconocimiento al generar mejores derechos para los trabajadores, protección en áreas de la salud, se crean los CAS (contratos administrativos de servicios) con la finalidad de dar solución a la problemática cada vez más agudizada de los contratos por servicios no personales, como una forma de dar solución a las restricciones presupuestales que se aplicaron para la contratación de personal en los regímenes generales, en el entendido de ahorro en costos y sobrecostos laborales y convenir a un modelo de negociación flexible; (3) Contratos de Servicios No Personales, refirió Beltrán (2013) que la referencia se remonta a contratos en el marco del D. S. N. ${ }^{\circ}$ 065-85-PCM, Reglamento Único de Adquisiciones, para el Suministro de Bienes y Prestaciones de Servicios No Personales del Estado; junio de 2008, se publica el Decreto Legislativo № 1017 que aprueba la Ley de Contrataciones del Estado, y crea el Organismo Supervisor de las Contrataciones del EstadoOSCE; enero del 2016 la OSCE informa la vigencia de la Ley $\mathrm{N}^{\circ} 30225$ - Ley de Contrataciones del Estado - LCE y su reglamento aprobado mediante Decreto Supremo $N^{\circ} 350$ 2015-EF.

Se incorpora un nuevo concepto, la Nueva Gestión Pública, pasa a ser un instrumento teórico o un enfoque diferente, que tiene implicancia gravitante en la gestión del personal, en ese sentido Ramírez (2012) refirió que se busca la instauración de una gestión efectiva, es decir, una gestión que cubra las carencias frecuentes de los habitantes al mínimo precio viable. Casas (2011), soslayó que la percibe como una secuencia perenne de perfeccionamiento con la finalidad de que el Estado responda a las perspectivas de los ciudadanos. Las justificaciones para implementar dicha Política fue asumir como deficiente e incorrecto el manejo y la administración del personal, que se sustentaba en la convivencia de desiguales regímenes laborales, la inexistencia de un marco laboral general y una rigidez normativa que contrastaba con la flexibilidad necesaria para atender las insuficiencias del colaborador de la administración pública dado la heterogeneidad de ellas; flexibilizar los procesos para incorporar y desligar colaboradores a plazo fijo a condiciones de contratos de naturaleza temporal; en esa línea se emite el D. S. No 004-2013 donde se dispone el "Servicio Civil Meritocrático" definido como paquete de normas colectivas que articula y gestiona a servidores del Estado, mediando entre los derechos sociales y los derechos de los colaboradores, siendo su finalidad el servicio al poblador, dichas acciones es confiada a la "Autoridad Nacional del Servicio Civil" (SERVIR).

Se planteó como problema general: ¿Cómo es el desempeño laboral e intención de rotación por régimen laboral de los trabajadores, Unidad de Recursos Humanos de la DRELM, periodo 2018?, como problemas específicos: ¿Cómo es el desempeño laboral de los trabajadores de la Unidad de Recursos Humanos de DRELM, 2018 en el régimen laboral del Decreto Legislativo 276?; ¿Cómo es el desempeño laboral de los trabajadores de la Unida de Recursos Humanos de la DRELM, 2018, en el régimen laboral del Decreto Legislativo 1057 CAS?;

Esta obra se comparte bajo la licencia Creative Common Atribución-No Comercial 4.0 International (CC BY-NC 4.0) Revista de la Universidad Internacional del Ecuador. URL: https://www.uide.edu.ec/ 
¿Cómo es el desempeño laboral de los trabajadores de la Unidad de Recursos Humanos de la DRELM, 2018, en el régimen de servicios no personales D. L. 30225?; ¿Cómo es la intención de rotación en los trabajadores de la Unidad de recursos Humanos de la DRELM, 2018, en el régimen laboral del Decreto Legislativo 276?; ¿Cómo es la intención de rotación en los trabajadores de la Unidad de Recursos Humanos de la DRELM, 2108, en el régimen laboral del Decreto Legislativo 1057 - CAS?; y finalmente, ¿Cómo es la intención de rotación de los trabajadores de la Unidad de Recursos Humanos de la DRELM, periodo 2108, en el régimen de servicios no personales D. L. 30225?

Estas preguntas generaron sendos objetivos que buscaban responderlas: Objetivo general: Analizar el desempeño laboral e intención de rotación por régimen laboral de los trabajadores, Unidad de Recursos Humanos de la DRELM, periodo 2018. objetivos específicos: Analizar el desempeño laboral de los trabajadores de la Unidad de Recursos Humanos de DRELM, 2018 en el régimen laboral del Decreto Legislativo 276; Analizar el desempeño laboral de los trabajadores de la Unida de Recursos Humanos de la DRELM, 2018, en el régimen laboral del Decreto Legislativo 1057 CAS; Analizar el desempeño laboral de los trabajadores de la Unidad de Recursos Humanos de la DRELM, 2018, en el régimen de servicios no personales D. L. 30225; Analizar la intención de rotación de los trabajadores de la Unidad de Recursos Humanos de la DRELM, periodo 2018, en el régimen del Decreto Legislativo 276; Analizar la intención de rotación de personal en los trabajadores de la Unidad de Recursos Humanos de la DRELM, 2018, en el régimen laboral del Decreto Legislativo 1057 - CAS; y, Analizar la intención de rotación del personal de los trabajadores de la Unidad de Recursos Humanos de la DRELM, 2108, en el régimen de servicios no personales D. L. 30225.

\section{Método}

Esta investigación fue de enfoque cualitativo, paradigma interpretativo, utilizando el estudio de caso y utilizando el análisis inductivo, la selección de la muestra fue deliberada e intencional de acuerdo con las limitaciones de los investigadores (Chaverra, Gaviria, y González, 2019; Hernández, Fernández y Baptista, 2014; Martínez, 1987; Martínez, 2011; Naupas et al., 2014; Quecedo y Castaño, 2002; Rodríguez, Gil y García, 1996; Ricoy, 2006; Yin, 1994)

Este trabajo se realizó en la Dirección Regional de Educación de Lima Metropolitana DRELM, que tiene como objetivo la gestión educativa descentralizada y rectora en Lima Metropolitana promoviendo y asegurando servicios educativos de calidad con equidad; dentro de esta instancia estatal se realizó las entrevistas en la unidad de recursos humanos - Dirección Regional de Educación de Lima Metropolitana periodo 2018. Balcázar et al., (2013), refirió que la zona en el cual el investigador o estudioso consigue cómodo acceso, donde se cimienta buena correlación con los sujetos y consigue referencias claramente afines al estudio; el periodo de análisis el año2018.

La selección se hace de acuerdo a como concuerdan a juicios o caracteres definidos por el científico, pudiendo ser una sola persona o institución como caso. Para esta investigación fueron: a) Funcionario Público: gerente de planeamiento y presupuesto; jefe de URH de la DRELM. Son 
dos personas, todos adultos de sexo masculino, con más de 05 años de experiencia en gestión pública. b) Servidores Públicos: Coordinadores y especialistas de la Unidad de Recursos Humanos que desarrollan sus actividades como supervisor, especialista de desarrollo de personas, especialista de planillas y especialista administrativo, siendo cuatro personas, todos adultos, dos de sexo femenino y dos de sexo masculino, con más de 05 años de experiencia en gestión pública de los cuales pertenecen al D.L. 276, al D. L. 1057 - CAS en actividad. En la tabla que sigue se muestra la codificación axial de los participantes en la investigación.

\section{Tabla 1}

Codificación de los sujetos participantes en la investigación

\begin{tabular}{lll}
\hline Sujetos & Letras & Código \\
\hline Gerente & G & G1, \\
Jefe de Unidad & J & J1 \\
Servidores & S & S1, S2, S3, S4,
\end{tabular}

Nota: Participantes involucrados en la investigación (2020)

Como técnicas de levantamiento de información se utilizaron la observación, la revisión documental y la entrevista (Ander-Egg, 2014; Baena, 1985; Ruiz, 2012). Los instrumentos utilizados fueron: una matriz de revisión o análisis documental y una guía de entrevista estructurada a profundidad de elaboración de los investigadores conformada por 16 preguntas abiertas, para un tiempo de respuesta de veinte minutos para los diversos participantes, previamente seleccionados ( Acevedo y López, 1986; Hernández, et al, 2014). En las tablas 1 y 2 se puede observar la codificación de los sujetos participantes en la investigación y la matriz de categorización de las variables del estudio.

\section{Tabla 2}

Matriz de Categorización del desempeño laboral e intención de rotación por régimen laboral

\begin{tabular}{lcc}
\hline \multicolumn{1}{c}{ Categoría } & Sub categoría & \multicolumn{1}{c}{ Indicadores } \\
\hline $\mathrm{C} 1$ & & C.1.1.1 Conocimiento \\
$\begin{array}{l}\text { Desempeño } \\
\text { laboral }\end{array}$ & C.1.1 Competencia &
\end{tabular}

$\begin{array}{ll}\text { C.1.2 Logro de metas } & \text { C.1.2.1 El individuo } \\ & \begin{array}{l}\text { C.1.2.2 Aspectos financieros } \\ \text { C.1.2.3 Posicionamiento institucional }\end{array} \\ \begin{array}{l}\text { C.1.3 Potencial de } \\ \text { mejoramiento }\end{array} & \text { C.1.3.1 Fines organizacionales } \\ & \text { C.1.3.2 Fortalecimiento de procesos }\end{array}$

C.1.1.2 Destrezas

C.1.1.3 Modos de comportamiento

C.1.2.2 Aspectos financieros

C.1.2.3 Posicionamiento institucional

C.1.3.2 Fortalecimiento de procesos 


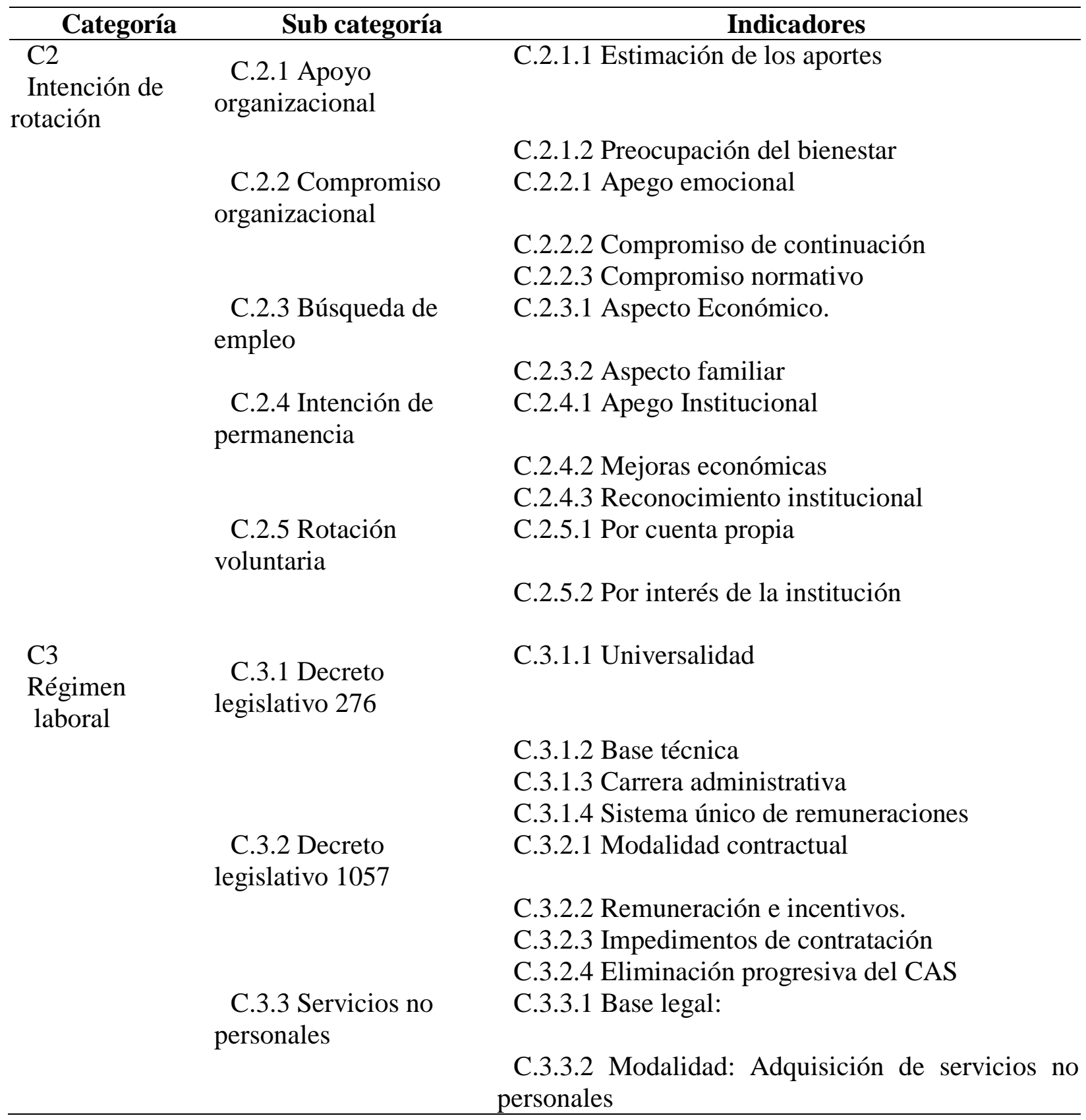

Nota: Categorización del desempeño laboral e intención de rotación por régimen laboral.

La técnica análisis, formulado mediante preguntas provenientes de las categorías, subcategorías e indicadores, siendo estas en forma clara, breve y precisas para su mejor entendimiento por los entrevistado; asimismo, ordenar los datos recopilados, posterior a la codificación para una mejor comprensión, estudio, análisis y difusión para esta investigación. Como tratamiento de lo moral, los conceptos éticos son por lo general y, ante todo, filosofía práctica, cuyo accionar no es el resolver conflictos, por el contrario, es plantearlos. La metodología del estudio cualitativo tiene muchas semejanzas con los aspectos éticos de la exploración convencional, el que podríamos interpretar la correlación entre la ciencia con los 
valores de verdad y justicia que en el presente estudio se aplica correctamente y acorde a los principios éticos y morales.

\section{Resultados}

Se realizaron seis entrevistas a distintos participantes, la información obtenida fue transcrita para luego desarrollar la categorización en el formato correspondiente para posteriormente realizar la codificación y matriz de triangulación de los datos obtenidos; a continuación se exponen las respuestas obtenidas de acuerdo a los objetivos planteados en este estudio. En la presente investigación, el análisis de los resultados se presenta como resultado a la triangulación (es una pericia donde se emplean tres o más aspectos o diferentes concurrentes, o varias fuentes de datos) de las respuestas de las entrevistas aplicadas a los participantes seleccionados, cuyos resultados generales interpretados se exponen a continuación:

Objetivo específico 1: Analizar el desempeño laboral de los trabajadores de la Unidad de Recursos Humanos (URH) de DRELM 2018 en el régimen laboral del Decreto Legislativo 276. Para responder a este objetivo se realizaron las siguientes preguntas:

1. $\quad$ En su opinión ¿Los trabajadores de la URH de la DRELM cuentan con los conocimientos y destrezas necesarias para el desempeño laboral? ¿Por qué? ¿Qué sugiere? Los entrevistados manifestaron que el personal que se incorpora a la DRELM se ubica en algunos cuatro posibles casos: a. Es su primer trabajo en el sector público y no tiene conocimiento ni experiencia. b. Es su segundo trabajo en el sector público y se encuentra en proceso de conocimiento y poca experiencia. c. Es más, es su tercer trabajo en el sector público y tiene conocimiento y experiencia, y d. Es su primer trabajo en el sector público; pero, tiene experiencia en el sector privado. En estos casos el personal enfrenta su incorporación con ciertas destrezas y conocimientos teóricos y/o prácticos para su desempeño laboral, sin embargo, la cultura organizacional no necesariamente es la misma. No existió un adecuado plan de desarrollo de capacidades ni el interés propio por los servidores nombrados del D. L. 276, que les permita evolucionar al mismo ritmo que la gestión pública, siendo deseable el desempeño laboral.

2. En su opinión ¿Los trabajadores de la URH de la DRELM manifiestan modos de comportamiento adecuados para su desempeño laboral? ¿Por qué? ¿Qué sugiere? Los entrevistados manifestaron que el personal se incorpora a la DRELM a través de diferentes normativas: a. Personal nombrado de la Ley 276; b. Personal contratado desde la Ley 276; c. Personal contratado CAS; d. Servicios de terceros. En ese contexto los comportamientos son disimiles y el avance no es igual en cada caso, porque cada quien adopta un comportamiento de acuerdo a la normativa y su contexto remunerativo, lo que no permite un desempeño laboral adecuado; buscan permanecer en sus puestos generando modos inadecuados de comportamiento, pese a estas limitaciones, se esfuerzan por desempeñarse adecuadamente de acuerdo a sus funciones.

Finalmente, los entrevistados manifestaron en relación al desempeño laboral de los trabajadores del régimen 276 es lo deseable; si bien, el personal enfrenta su incorporación con 
ciertas destrezas, conocimientos teóricos y/o prácticos y que siempre la cultura organizacional no necesariamente es la misma, la inexistencia de un adecuado plan de desarrollo de capacidades y su propio interés no les permite evolucionar al mismo ritmo; adicionalmente, se incorporan a la DRELM con diferentes normativas; los comportamientos son disimiles y el avance no es igual, porque cada quien adopta un comportamiento de acuerdo a su contexto remunerativo lo que no permite un desempeño laboral adecuado, pese a estas limitaciones, se esfuerzan por desempeñarse adecuadamente de acuerdo a sus funciones.

Objetivo específico 2: Analizar el desempeño laboral de los trabajadores de la URH de la DRELM 2018, en el régimen laboral del Decreto Legislativo 1057 CAS. Para responder a este objetivo se realizaron las siguientes preguntas:

3. En su opinión ¿Los trabajadores CAS de la URH de la DRELM cumplen con lograr las metas planteadas por la institución de forma individual o colectiva? ¿Qué sugiere? Los entrevistados manifestaron que, al no estar establecidos los indicadores de medición y al obtener resultados positivos no permite su sistematización, desconociendo si el trabajo individual o colectivo son paralelos o cada cual va por su lado; se debe establecer indicadores para cada puesto, que induzca al personal a ir por estándares uniformes. De forma individual si se cumplen, porque hay una ratio que mide el cumplimiento de metas y son presionados para lograr sin importar horas adicionales de su jornada, se indicó que ambas cumplen. La institución viene realizando capacitaciones a servidores CAS y CAP, en la búsqueda de la mejora continua, que ayude a cumplir con las metas planteadas, finalmente para el personal contratado por el D. L. 1057, sus conocimientos y destrezas son superiores, permitiendo un adecuado desempeño laboral.

4. $\quad$ En su opinión ¿Usted considera que los trabajadores CAS de la URH de la DRELM contribuyen al logro de las metas financieras y al posicionamiento institucional? ¿Por qué? ¿Qué sugiere? Los entrevistados manifestaron que el logro de metas financieras y el posicionamiento institucional se evidencia a través de la consulta amigable del MEF, los cuales deben ser siempre superiores al $95 \%$ en ejecución. En general los CAS contribuyeron al logro de las metas financieras y al posicionamiento institucional, lo que ocurre es que no se mide el aporte individual, solo se mide aquellos que tienen indicadores por gestión y no en todos sus procedimientos desde el nivel más bajo hasta el más alto.

Finalmente, los entrevistados manifestaron que, al no contar con indicadores de medición y los resultados positivos no son sistematizados, desconociendo si el trabajo individual o colectivo son paralelos o cada cual va por su lado; de forma individual si se cumplen, porque hay una ratio que mide el cumplimiento. Por otro lado, el logro de metas financieras y el posicionamiento institucional se evidencia a través de la consulta amigable del MEF que debe ser superiores al 95\% en ejecución; los CAS contribuyeron al logro de dichas metas, pese que solo se mide aquellos que tienen indicadores por gestión y no en todos sus procedimientos. Consecuentemente, los CAS si contribuyen en gran medida al logro de metas, por consiguiente, es óptimo el desempeño laboral de los trabajadores del régimen laboral 1057 - CAS. 
Objetivo específico 3: Analizar el desempeño laboral de los trabajadores de la URH de la DRELM 2018, en el régimen de servicios no personales D. L. 30225. Para responder a este objetivo se realizaron las siguientes preguntas:

5. En su opinión ¿la URH de la DRELM cuenta con el recurso humano que le permita mejorar los fines y metas institucionales? ¿Cómo? ¿Qué sugiere? Los entrevistados manifestaron que se tiene la idea errónea que, a mayor cantidad de personal, se tiene una Unidad más sólida, nos encontraos retrasados en atender los diversos temas del personal, se hace necesario contratar servicios no personales para el desembalse de expedientes sin respuesta al identificar los principales procesos que generan mayor demanda. En ese sentido a pesar de las dificultades en materia de recurso humano, si, se cuenta con el recurso humano capaz de optimizar la calidad de la prestación, contribuyendo al logro de fines y metas institucionales.

6. $\quad$ En su opinión ¿la URH de la DRELM cuenta con el potencial que le permita fortalecer y mejorar los procesos y procedimientos? ¿Por qué? ¿Qué sugiere? Los entrevistados manifestaron que no todas las entidades cuentan con el perfil de profesionales para optimizar, identificar el potencial y mejorar los procesos y procedimientos. Es decir, falta fortalecer con recursos humanos especializados, que conozcan no solo la cultura organizacional del sector sino de los regímenes laborales que administra la URH. Si, se cuenta con potencial adecuado, dejando de lado las individualidades, mayor identificación institucional, personal calificado, comprometido y reconocido, que permitirá fortalecer, mejorar procesos y procedimientos a fin de no generar contrataciones por terceros, que no necesariamente son especialista. La institución no cuenta con un proceso de adiestramiento, estimación del desempeño laboral, conocer las necesidades de los equipos de trabajo y los sistemas de control y monitoreo.

Finalmente, los entrevistados manifestaron que se tiene la idea errónea que, a mayor cantidad de personal, se tiene una Unidad más sólida, lo cual está lejos de la realidad, haciéndose necesario contratar servicios no personales para el desembalse de los expedientes no atendidos, la entidad si cuentan con el potencial adecuado, que permita mejorar los procesos y procedimientos; asimismo, no cuenta con un proceso de adiestramiento, estimación del desempeño laboral, de un sistema de control y monitoreo de personal, a fin de no generar contrataciones por terceros, que no necesariamente son especialistas, por consiguiente, el desempeño laboral de los trabajadores de la Unidad de Recursos Humanos de la DRELM 2018, en el régimen de servicios no personales D. L. 30225, no es determinante ni óptimo.

Objetivo específico 4: Analizar la intención de rotación de los trabajadores de la URH de la DRELM 2018, en el régimen del Decreto Legislativo 276. Para responder a este objetivo se realizaron las siguientes preguntas:

7. En su opinión ¿La institución brinda el apoyo institucional recepcionando o estimando los aportes de los trabajadores nombrados la URH de la DRELM? ¿Cómo? ¿Que sugiere? Los entrevistados manifestaron que lo ideal es tener una autoridad que escuche y atienda los aportes, siendo la unidad quien debe valorar el aporte del personal en armonía con el marco normativo. Es necesario reuniones de trabajo periódicas que identifique los principales problemas, los aportes del personal nombrado y percibir las necesidades e intenciones de rotación interna o externa. En ese sentido, no siempre se presta atención a los aportes de los 
trabajadores que permita eliminar los cuellos de botella y las posibles intensiones de rotación a otros regímenes o externas, debido a la idea errónea de percibir que dichos aportes son negativos y contra la institución; las funciones que cumple el personal en diversos niveles conforme a su formación académica, busca resolver las acciones administrativas, más allá de si la institución brinda el apoyo institucional recepcionando o estimando sus aportes.

7. En su opinión ¿La institución brinda el apoyo y muestra su preocupación por el bienestar o la intensión de rotación de los trabajadores de la URH de la DRELM? ¿Cómo? ¿Que sugiere? Los entrevistados manifestaron que la institución apoya con capacitaciones para ciertas personas, se viene avanzando con la mejora del bienestar de los trabajadores, como una forma de compensar los desniveles remunerativos con otros regímenes laborales; es una preocupación constante la intención de rotación de los trabajadores nombrados, hay disconformidad por las remuneraciones, esconden su intención de cambiar a otro régimen laboral, manteniéndose latente, nadie habla abiertamente, de forma personal y reservada hacen saber su intención. Se trabajó de forma individual a fin de reconocer el desempeño laboral y nivelar las remuneraciones dentro de la flexibilidad de las normas. Asimismo, la prioridad de la institución en hacer cumplir las funciones en los plazos establecidos, no le permite promover una empatía con el trabajador.

Finamente, los entrevistados manifestaron que lo ideal es tener una autoridad que escuche y atienda este Tipo de aportes, que valore el aporte del personal en armonía con el marco normativo. Se entiende que no se recepcionan o estima los aportes, no existe el interés por conocer sus necesidades, sus intenciones de rotación o traslado, debido a la idea errónea de que dichos aportes todos son negativos y contra la institución. Es una preocupación constante la intención de rotación de los trabajadores nombrados, hay disconformidad por las remuneraciones, esconden su intención de cambiar a otro régimen laboral, la intención está latente, nadie habla abiertamente, de forma personal y reservada hacen saber su intención. Notándose una clara intención de rotación interna o externa de los trabajadores del Régimen laboral del D. L. 276.

Objetivo específico 5: Analizar la intención de rotación de personal en los trabajadores de la URH de la DRELM 2108, en el régimen laboral del Decreto Legislativo 1057 - CAS. Para responder a este objetivo se realizaron las siguientes preguntas:

9. En su opinión ¿Los trabajadores CAS de la URH de la DRELM muestran identificación institucional y compromiso por conocer y cumplir el marco normativo? ¿Por qué? ¿Que sugiere? Los entrevistados manifestaron que existe personal CAS que muestra mayor disposición y actitud que los demás, aportan en el marco de sus funciones asignadas y otros buscan mayores retos que logre la mejora de la Unidad. Se hace la distinción entre identificación y nivel de compromiso del personal nombrado y personal contratado, para el primero es subjetivo, el sentido de pertenencia es el generador de derechos y no de obligaciones, para el segundo, el nivel de identificación y compromiso está directamente relacionado en el alcanzar niveles aprobatorios de eficiencia y eficacia que coadyuven a la realización de metas y objetivos de la entidad en base a resultados, son profesionales que asumen el compromiso ético y deseo de contribuir, consecuentemente los trabajadores CAS de la Unidad de Recursos Humanos de la DRELM muestran identificación institucional y compromiso por conocer y cumplir el marco normativo. 
10. En su opinión ¿Los trabajadores de la URH de la DRELM muestran a menudo la intención de buscar empleo que le permita mejorar su estatus económico o familiar? ¿Por qué? ¿Que sugiere? Los entrevistados manifestaron que hasta mitad del 2014 la rotación de personal era permanente, debido al bajo nivel remunerativo; se capacitaban e inmediatamente se ponían a la búsqueda de nuevas oportunidades y en algunos casos eran contratados por otras entidades. La distinción es en base a los grupos plenamente identificados, el personal nombrado y el contratado, la deserción y la volatilidad está en el segundo grupo, no existe una estandarización remunerativa para el nivel profesional. Para los servidores CAS, sobre todo los que conocen su potencial, muestran a menudo la intención de buscar empleo que le permita mejorar su estatus económico o familiar, en otros casos dicha intensión es porque no se siente reconocidos, no hay futuro profesional o línea de carrera.

11. En su opinión ¿Los trabajadores de la URH de la DRELM muestran a menudo la intención de permanecer en la institución por su identificación con la institución, por mejoras económicas o porque existe un reconocimiento institucional? ¿Por qué? ¿Que sugiere? Los entrevistados manifestaron que en los últimos años los trabajadores de la unidad manifiestan su intención de permanecer laborando, pero siempre el tema de buscar mejoras económicas es un detonante para renunciar e irse a otra institución; en otros casos, profesionales que no son adecuadamente ubicados en cargos que les permita mostrar un mejor desempeño o rendimiento, el personal contratado bajo cualquier régimen laboral, emigra ante una mejora remunerativa constituyéndose en una de las principales razones que afecta la identificación institucional. Pese que no existe reconocimiento institucional los CAS; si, continúan en el puesto, es por el servicio que se presta al administrado y otros por un tema de costumbre, en su mayoría permanecen en la institución por lograr la estabilidad laboral.

12. En su opinión ¿Los trabajadores de la URH de la DRELM muestran su voluntad de rotación interna o externa de la institución por cuenta propia o por interés institucional? ¿Por qué? ¿Que sugiere? Los entrevistados manifestaron que en ciertos casos hay trabajadores que muestran su voluntad de rotar internamente, porque siente que desean asumir otros retos laborales, mostrar su capacidad y buscar nuevos horizontes. Se dan por cuenta propia y otros por propuestas de algunos jefes. Ello desequilibra los equipos cuando estos han logrado articularse y muestran una performance regular. El personal CAS no tiene problemas en rotar por interés institucional. La rotación interna o externa es por interés personal, siempre en busca de mejoras.

Finalmente, los entrevistados manifestaron que existe personal CAS que siempre muestra mayor disposición y actitud, aportan en el marco de sus funciones asignadas y otros buscan mayores retos que logre mejorar la Unidad, el nivel de identificación y compromiso que está directamente relacionado con alcanzar niveles aprobatorios de eficiencia, eficacia y compromiso ético. La deserción y la volatilidad está en los CAS, por cuanto no existe una estandarización remunerativa, muestran a menudo la intención de buscar empleo que le permita mejorar su estatus económico o familiar, siempre el buscar mejoras económicas es un detonante para renunciar, siendo una de las razones que afecta la identificación institucional. La rotación interna o externa de los trabajadores de la Unidad de Recursos Humanos de la DRELM 2108, en el régimen laboral del Decreto Legislativo 1057 - CAS es por interés personal, siempre en busca de mejoras económicas y familiares. 
Objetivo específico 6: Analizar la intención de rotación del personal de los trabajadores de la URH de la DRELM, en el Régimen de servicios no personales D. L. 30225. Para responder a este objetivo se realizaron las siguientes preguntas:

13. En su opinión ¿Los trabajadores de la URH de la DRELM conocen los regímenes laborales y el sistema remunerativo, que regula su relación contractual con la DRELM? ¿Por qué? ¿Que sugiere? Los entrevistados manifestaron que algunos están al tanto y claros sobre los diferentes regímenes laborales que administra la entidad, por encontrarse absorbidos por el día a día y no tengan interés o tiempo para conocer a profundidad esos temas, lo que sí es seguro que casi la totalidad de los mismos conocen el régimen y las características de su contratación personal, con ello conocen sus derechos y deberes. La mayoría de los casos, muestran su preocupación por su retribución económica, se ha dado algunos casos que han laborado por más de 25 años en calidad de nombrados renunciado a su plaza para optar por una plaza CAS o por contratos de servicios no personales, por mejoras remunerativas, estas son las distorsiones que se generan por la coexistencia de regímenes laborales diversos con sus sistemas remunerativos en la DRELM.

14. En su opinión ¿Los trabajadores de la URH de la DRELM se sienten protegidos o desprotegidos por la modalidad contractual o por las leyes laborales, en relación a sus derechos laborales? ¿Por qué? ¿Que sugiere? Los entrevistados manifestaron que las diferentes leyes laborales siempre demandaran mejoras respecto de sus derechos laborales, que muchas veces no son atendidos por qué no se visibiliza adecuadamente desde la propia Unidad. Respecto al personal contratados por los diferentes regímenes laborales, se siente desprotegidos, visto que en la práctica no les asiste ningún derecho respecto de la seguridad contractual de permanencia como desarrollo de una carrera profesional, no existe un trato igualitario, los colaboradores por servicios no personales tampoco tienen un beneficio adicional, sus órdenes de servicio tiene vigencia periódica hasta la entrega del producto, pudiendo ser renovado como no también.

15. En su opinión ¿la institución cuenta con una política de gestión de los recursos humanos, que permita la regulación contractual por un solo régimen laboral? ¿Por qué? ¿Que sugiere? Los entrevistados manifestaron que la institución como órgano desconcentrado del Minedu encargada de regular los diferentes regímenes, los cuales no están siendo atendidos. Por otro lado, la implementación del Régimen del Servicio Civil Ley $\mathrm{N}^{\circ}$ 30057, sería una adecuada alternativa al acceso de forma meritocrática; por consiguiente, no existe política de gestión de recursos humanos con regulación contractual por un solo régimen laboral, existiendo personal CAP, CAS, y por servicios no personales, con diferentes remuneraciones para igual cargos, puestos o escalafón, existe preferencias salariales, sueldos por encima de lo que corresponde, sin equidad y maltrato a los colaboradores.

16. En su opinión ¿Por qué la autoridad realiza la contratación de servicios de terceros para actividades permanentes de los cargos de la URH de la DRELM? Los entrevistados manifestaron que, tiene que ver con el dimensionamiento institucional, donde no es atendido desde un nivel mínimo para su funcionamiento, a pesar del pedido reiterado del presupuesto, ello motiva que algunas funciones no realizadas son cubiertas por personal contrató bajo la modalidad de servicios de terceros que evitan un embalse de expedientes en trámite, es una 
alternativa que puede generar ciertas condiciones al personal, pero es un mecanismo utilizado, pese que hay una imposibilidad normativa para la contratación bajo esta modalidad en plazas de prestación permanente, otras son de apoyo a la institución que no tiene un perfil especifico, sus funciones no generan responsabilidad, no son protegidos por las normas laborales y no cuentan con beneficios, siendo utilizadas para "rotar" a un contrato administrativo de Servicios - CAS.

Finalmente, los entrevistados manifestaron que algunos están al tanto y claros sobre los diferentes regímenes laborales que administra la entidad, lo que sí es seguro que casi la totalidad de los mismos conocen el régimen y las características de su contratación personal. En estos regímenes laborales hay gran diferencia salarial entre nombrados, CAS y contratos por servicios no personales. Por otro lado, las diferentes leyes laborales siempre demandaran mejoras respecto de sus derechos laborales que no son atendidos, se siente desprotegidos, respecto de la seguridad contractual, el desarrollo de una carrera profesional, no existe un trato igualitario, ni derechos laborales que ampare al colaborador, ni beneficio adicional a los contratos de servicios no personales, hay una imposibilidad normativa para la contratación bajo esta modalidad en plazas de prestación permanente en los cargos, puestos o escalafón, otras no tienen perfil especifico, no generan responsabilidad no cuentan con beneficios, siendo utilizadas para "rotar" a un contrato administrativo de Servicios - CAS.

Objetivo general: analizar el desempeño laboral y la intención de rotación por régimen laboral de los trabajadores, URH de la DRELM 2018, los participantes de la entrevista señalaron en forma general que el desempeño laboral de los trabajadores de la URH de la DRELM en el régimen laboral del D. L. 276 es deseable, dada las condiciones de su incorporan en los diferentes regímenes laborales, generando comportamientos disimiles de acuerdos a su régimen y contexto remunerativo, sus destrezas y sus conocimientos teóricos y/o prácticos, pese no haber existido un adecuado plan de desarrollo de capacidades, manifiestan modos de comportamiento adecuados con ciertas limitaciones. Asimismo indicaron que para los CAS, no está establecido los indicadores de medición y los resultados positivos no pueden ser sistematizados, desconociendo si el trabajo individual o colectivo son paralelos o cada cual va por su lado; el logro de metas financieras y el posicionamiento institucional se evidencia a través de la consulta amigable del MEF en la ejecución, pese que no se mide el aporte individual, por consiguiente indican que es óptimo el desempeño laboral de los trabajadores del régimen laboral 1057 - CAS.

A su vez indicaron que, se tiene la idea errónea que a mayor cantidad de personal, se tiene una unidad más sólida, la realidad lo contradice, por cuanto, se hace necesario la contratación de especialistas por contratos no personales para el desembalse documentario; pese que la entidad cuenta con el potencial humano que mejore los procesos y procedimientos; sin embargo, no cuenta con sistemas de control y monitoreo de los avances del trabajo, lo que generar contrataciones de terceros, que no necesariamente son especialistas, no siendo determinante ni optimo el desempeño laboral de los trabajadores del régimen de servicios no personales D. L. 30225. Por otra parte indicaron que, lo ideal es tener una autoridad que escuche y atienda los aportes en armonía con las normas, identifique las necesidades e intención de rotación del trabajador nombrado, pese a no contar con ello, busca resolver las acciones administrativas, más allá, de si cuenta o no con el apoyo institucional; hay avances en mejorar el bienestar del trabajador, como una forma de compensar los desniveles remunerativos entre 
regímenes laborales, encontrándose latente la intención de rotación interna de los trabajadores del régimen laboral 276.

De igual modo, existen trabajadores CAS que muestra mayor disposición y actitud que los demás, aportan en el marco de sus funciones asignadas y otros buscan mayores retos para la mejora de la Unidad, muestran identificación institucional y compromiso por conocer y cumplir el marco normativo, pese a ello, la deserción y la volatilidad está en ellos, por consiguiente, los trabajadores del régimen laboral 1057 - CAS muestran a menudo la intención de buscar empleo que le permita mejorar su estatus económico o familiar. Del mismo modo, conocen los diferentes regímenes laborales que administra la entidad, lo que sí es seguro que casi la totalidad de los mismos conocen el régimen y las características de su contratación personal; pese a ello, se siente desprotegidos, sienten que en la práctica no les asiste ningún derecho, no perciben permanencia en el tiempo, las diferencias salarias son abismales, no existe un trato igualitario; los contratados por servicios no personales, no tienen un beneficio adicional, no tienen perfil especifico, no generan responsabilidad, no cuentan con beneficios, existe una imposibilidad normativa para la contratación por el régimen de servicios no personales D. L. 30225 en plazas de prestación permanente en los cargos, puestos o escalafón, siendo utilizadas para "rotar" a un contrato administrativo de servicios - CAS.

Análisis e interpretación de las categorías emergentes: luego de la aplicación de la entrevista a profundidad se obtuvieron las siguientes categorías, las tres primeras categorías consideradas antes del diagnóstico, con la aplicación del instrumento se llegaron a identificar dos últimas Régimen Servir y Cultura organizacional.

La categoría Régimen Servir, lo definió el MINJUS (2017) Ley Nº 30057 - Ley de Servicio Civil es un significativo progreso en la innovación de la administración pública, por cuanto formula el propósito del Estado de contar con un régimen único y propio para los individuos que proporcionan sus servicios en el gobierno público, denominado servicio civil. Dicha ley apunta a proteger el principio de mérito en la gestión de las personas, con el objetivo de tener personal cualificado e idóneo para el desarrollo de los puestos del Estado. Cortez y Prieto (2015) refirieron a los individuos que prestan servicio al Estado, asimismo a las normas institucionales que articula y gestiona a dichas personas. Los entrevistados manifestaron que la institución debería implementar el régimen servir, como una alternativa adecuada que permitirá acceso de forma meritocrático a una plaza en el servicio civil, con el propósito de terminar con la proliferación de regímenes.

Cultura organizacional, según Robbins y Judge (2009) refirieron, la cultura no es un fenómeno apartado, que, influye en la creación, en los efectos, en las personas, en los equipos, en la minuciosidad, agresividad, estabilidad, estructura, en el liderazgo, organización y ambiente. Asimismo, Martínez (2010) puntualizo que, la cultura representa un procedimiento de trascendencia y estimación que incita al conjunto social a precisar la misión y, es un aspecto intelectual del medio artificial instituido por los entes como resultado de su coexistencia social. Por lo que se refirió Góngora, Reija y Larrivey (2013) cultura en valores, normas, creencias, artefactos y conjeturas elementales que persisten a largo plazo y que, expresan las particularidades sobresalientes en la entidad y sus integrantes. Los entrevistados indicaron que la cultura organizacional de las entidades donde antes desarrollaron su experiencia laboral no es 
necesariamente igual a la cultura organizacional de la DRELM, generando un desfase de desconocimiento en su desempeño laboral.

\section{Conclusiones y Recomendaciones}

Dada las condiciones de incorporación en diferentes regímenes a generando conductas disimiles de acuerdos a su contexto remunerativo, sus destrezas y sus conocimientos teóricos y/o prácticos manifiestan comportamientos adecuados con ciertas limitaciones, siendo deseable el desempeño laboral de los trabajadores de la URH de la DRELM en el régimen laboral del D. L. 276. Se recomienda a la Unidad de Recursos Humanos de la DRELM, establecer un plan de inducción dirigido al colaborador que se incorpora a la institución, que permita, dar a conocer con información básica del movimiento, de la estructura, los cargos y evitar distorsiones en el desenvolvimiento de los trabajadores, evitar que la pérdida de tiempo y recursos intentando solucionar ciertas dudas; siendo la meta, que el colaborador se habitúe a la institución, a los compañeros de trabajo y directivos, asimismo de interiorizar la cultura, la historia, la misión, la visión, objetivos y políticas institucionales y del sector educación.

Para los CAS, no está establecido indicadores de control y los resultados no pueden ser sistematizados, desconociendo si el trabajo individual o colectivo son paralelos o cada cual va por su lado, pese que no se mide el aporte individual, si contribuyen al logro de metas financieras y al posicionamiento institucional, siendo óptimo el desempeño laboral de los trabajadores del régimen laboral 1057 - CAS. Se sugiere a la Unidad de Recursos Humanos de la DRELM, implementar de forma periódica la evaluación de desempeño laboral, como una tarea clave de la Unidad de Recursos Humanos en la gestión de personal por régimen laboral, que admita conocer las fortalezas y debilidades de los servidores, así como conocer sus necesidades y cómo influyen en la organización su intención de rotación, así mismo, sirva para la toma de decisiones.

Se tiene la idea errónea que, a mayor cantidad de personal una unidad más sólida, la realidad lo contradice, no se cuenta con sistemas de control y monitoreo de los avances del trabajo por lo que se recurre a contratos no personales de especialistas para el desembalse documentario, donde no necesariamente son especialistas, no siendo determinante ni optimo el desempeño laboral de los trabajadores del régimen de servicios no personales D. L. 30225. Se sugiere a la Unidad de Recursos Humanos de la DRELM, diseñar y elaborar un plan de rotación interna de los servidores nombrados (D. L. 276) y contratados por CAS (D. L. 1057), que tenga como insumo las necesidades objetivas de la institución, así como la disponibilidad de los servidores, como parte del convenio o pacto entre ellos y la institución.

Lo ideal es tener una autoridad que escuche y atienda los aportes en armonía con las normas, que identifique las necesidades e intención de rotación del trabajador nombrado, hay avances en mejorar el bienestar del trabajador, como una forma de compensar los desniveles remunerativos entre regímenes, encontrándose latente la intención de rotación interna de los trabajadores del régimen laboral 276. Se sugiere a la Unidad de Recursos Humanos de la 
DRELM, efectivizar el traslado de los trabajadores del régimen de CAS hacia la planilla del régimen general imperante en la institución (D. L. 276), hasta la implementación del Servicio Civil de la Ley 30057, a fin de que esta modalidad de contratación no siga desnaturalizado la esfera laboral, y que estos servidores CAS, desarrollen su trabajo de forma permanente, con beneficios con que cuenta otros trabajadores en el mismo puesto, cargo o escalafón.

Los CAS muestran mayor disposición y actitud, aportan en el marco de sus funciones y otros buscan mayores retos para la mejora de la Unidad, muestran identificación institucional y compromiso por conocer y cumplir el marco normativo, pese a ello, la deserción y la volatilidad está en ellos, los trabajadores del régimen laboral 1057 - CAS muestran la intención de buscar empleo que les permita mejoras económicas y familiar. Se recomienda a la Unidad de Recursos Humanos de la DRELM, evitar la contratación por servicios no personales para labores con finalidad permanente, considerando que este tipo de contrataciones es una prestación independiente, sin sujeción a jornada laboral ordinaria, la inexistencia de la subordinación con el empleador, no cuentan con beneficios laborales, con el fin de no vulnerar un contrato de carácter laboral y los derechos de los trabajadores.

\section{Referencias Bibliográficas}

Acevedo, M. y López, A. (1986). El proceso de la entrevista conceptos y modelos (3 ed.). México DF: LIMUSA. Recuperado de https://bit.ly/3dAyJhx

Baena, G. (1985) Manual para elaborar trabajos de investigación documental. Publisher: México: Edits. Mexicanos Unidos, 1985. diction/Format: Print book: Spanish: 4a ed.

Balcázar, P., González-Arratia, N., Gurrola, G. y Moysén, A. (2013). Investigación cualitativa. Ministerio de Educación. Recuperado de: https://bit.ly/3iMBobs

Beltrán, L. (2013). Problemática de la Existencia de Distintos Regímenes de Contratación de Personal En El Estado, [Tesis Maestría, Universidad Católica]. Recuperado de https://bit.ly/2Ba3o87

Bohlander, G. \& Snell, S. (2013). Human resources management. 14ta. Edition. Cengage Learning Editors S.A. México. Recuperado de https://bit.ly/3dyHrgb

Buitrago E., Constain, L. y Lemus L. (2016). Rotación laboral voluntaria en jóvenes profesionales de la generación millennials de la ciudad de Cali.

Campbell, J., Dunnete, M., Lawler, E., \& Weick, K. (1970). Managerial Behavior, Performance and Effectiveness. Nueva York: McGraw-Hill.

Campos, P., Gutiérrez, J. y Matzumura K. (2018). Rotação e performance no trabalho dos profissionais de enfermagem. Revista Cuidarte. Recuperado de: https://bit.ly/2YAmWeI.

Casas, C. (2011). Avances de la Reforma y Modernización del Estado en el Perú. Documento Preliminar. Recuperado de: https://bit.ly/2Z9HtHy

Castellanos, R. (2017) La coexistencia de regímenes laborales en las entidades públicas como causa de las diferencias y trato discriminatorio de los servidores públicos analizados en la red asistencial de Lambayeque EsSalud. [Tesis doctoral. Universidad Pedro Ruiz Gallo].

Castillo, J. et. Al. (2011). Compendio de derecho Laboral Peruano. Ediciones Caballero Bustamante. Lima Perú.

Esta obra se comparte bajo la licencia Creative Common Atribución-No Comercial 4.0 International (CC BY-NC 4.0) Revista de la Universidad Internacional del Ecuador. URL: https://www.uide.edu.ec/ 
CEPAL (2012). Flexibilización laboral en el Perú y reformas de la protección social asociadas: Un balance tras 20 años. Visión de desarrollo social, Santiago. Recuperado de: https://bit.ly/31L6WbI

Cepeda, R. (2017). Los regímenes laborales y su relación con el desempeño laboral de los trabajadores de la Fiscalía Especializada de Lavado de Activos y Pérdida de Dominio, 2017, [Tesis de Maestría, UCV]. Recuperado de: https://bit.ly/2ZeWV3S.

Chaverra, B., Gaviria, D. y González, E. (2019). El estudio de caso como alternativa metodológica en la investigación en educación física, deporte y actividad física. Conceptualización y aplicación. Recuperado de: https://bit.ly/3gCYkIo

Chávez, P. (2001). Flexibilidad en el mercado laboral: orígenes y concepto. Aportes, mayoagosto, año/vol. VI, número 017 Benemérita Universidad Autónoma de Puebla - Puebla, México. Recuperado de: https://bit.ly/2Z4GTus

Chiang, M. y San Martín, N. (2015), Análisis de la satisfacción y el desempeño laboral en los funcionarios de la municipalidad de Talcahuano, Cienc. Trab. Sep.-Dic; 17 [54]: 159165). Recuperado de: https://bit.ly/3fUlicG.

Chiavenato, I. (2011). Administración de Recursos Humanos. El capital humano de las organizaciones. 9na. Edición. McGraw-Hill Interamericana- Editores, S. A. DE C. V. México. Recuperado de: https://bit.ly/3jNLXLf

Chiavenato, I. (2014). Comportamiento organizacional (3ra. ed.). McGraw Hill. México.

Constitución Política del República del Perú (1979). Recuperado de: https://bit.ly/38Bxbmi

Constitución Política del República del Perú (1993). Recuperado de: https://bit.ly/3gCu514

Cortez, J. y Prieto, T. (2015). La reforma del servicio civil en el Perú: la profesionalización de la función pública, XX Congreso Internacional del CLAD sobre la Reforma del Estado y de la Administración Pública, Lima, Perú, 10-13 nov. 20. Recuperado de: https://bit.ly/3dFViBg.

Decreto Legislativo $\mathrm{N}^{\circ}$ 276/1984, 06 de marzo, Ley de bases de la carrera administrativa y de remuneraciones del sector público, Publicado el 26 de marzo de 1984. Recuperado de: https://bit.ly/3eEqLVI

Decreto Legislativo $\mathrm{N}^{\circ}$ 1057/2008, 26 de junio, Regula el régimen especial de contratación administrativa de servicios (2008). Diario oficial el peruano, pp. 374986. Recuperado de: https://bit.ly/3ecvQUe

Decreto Legislativo $\mathrm{N}^{\circ}$ 30225/2016, del 01 de enero, Ley de contrataciones del Estado y su reglamento. Lima - Perú.

Drucker, P. (2002). Managing in the Next Society. Published by Elsevier Ltd. Recuperado de: https://bit.ly/3gr3rLR

Eisenberger, R., Huntington, R., Hutchison, S. y Sowa, D. (1986). Perceived organizational support. Journal of Applied Psychology. Recuperado de: https://bit.ly/3e9J3wY

Espinal, J. (2008). La gestión de personal en la administración pública: Manual de consulta para funcionarios y servidores públicos. Ed. Centro de estudios gubernamentales, IPAC. Lima - Perú.

Fischbein, M., \& Ajzen, I. (1975). Belief, Attitude, Intention, and Behavior: An Introduction to Theory and Research. Reading, MA: Addison-Wesley. Recuperado de: https://bit.ly/3f8S239

Góngora, N., Reija, L. y Larrivey, M. (2013). La confianza organizacional en los institutos de investigación. Revistas UNL Pam. Recuperado de: https://bit.ly/385696z

Esta obra se comparte bajo la licencia Creative Common Atribución-No Comercial 4.0 International (CC BY-NC 4.0) 
Griffeth, R., Hom, P., \& Gaertner, S. (2000). A meta-analysis of antecedents and correlates of employee turnover: Update, moderator tests, and research implications for the next millennium. Journal of Management, 26 (3).

Gruezo, X. (2017). Análisis del desempeño laboral de la dirección de gestión de planificación y proyectos del gobierno autónomo descentralizado municipal del cantón rio verde, Esmeraldas, Ecuador, junio 2017. https://bit.ly/2ZgCMds

Hernández, R.; Fernández, C. y Baptista, M. (2014). Metodología de la investigación. Recuperado de: https://bit.ly/2WFtxTO

Herzberg, F., Mausner, B., \& Snydermann, B. (1959). Motivation at work. New York: Wiley The Theory of the two factors.

Hom, P., y Griffeth, R. (1991). Structural equations modeling test of a turnover theory: Crosssectional and longitudinal analyses. Journal of Applied Psychology, 76,350-366. http:// bit.ly/32XQ513

Ley $\mathrm{N}^{\circ} 30057 / 2013,03$ de julio, Ley del servicio civil. Publicado Diario Oficial el Peruano $\mathrm{N}^{\circ}$ 298, pp. 585. Recuperado de: https://bit.ly/2Zkol8C

Littlewood, H. (2006). Antecedentes de la Rotación Voluntaria de Personal, Investigación Administrativa, RIA 97, enero-junio, 2006, Escuela Superior de Comercio y Administración, Unidad Santo Tomás Distrito Federal, México. Recuperado de: https://bit.ly/2NAUpzA.

Maldonado, J., Monteza, H. y Rosales, D. (2018). El engagement y la intención de rotar de los trabajadores en las notarías de lima. [Universidad del Pacifico]. Recuperado de: https://bit.ly/2ZFCPQg

Martínez, F. (1987). El método inductivo. [Universidad Autónoma de Nuevo León]. Recuperado de https://bit.ly/38G8PIb.

Martínez, M. (2010). Relaciones entre cultura y desempeño organizacional en una muestra de empresas colombianas: reflexiones sobre la utilización del modelo de Denison. Cuadernos de Administración. 23 (40), 163-190. Recuperado de: https://bit.ly/3dDXUjd.

Maslow, A. (1943) Theory of human motivation. Recuperado de: file://C:/Users/MyPC/AppData/Local/Temp/1-52-PB.pdf

Meyer, J. \& Allen, N. (1991). A three-component conceptualization of organizational commitment. Human Resources Management Review.

McGregor, D. (1960). The human side of enterprise. McGraw-Hill: Recuperado de: https://bit.ly/2BFNP8r

Mobley, W. (1977). "Intermediate linkages in the relationship between job satisfaction and employee turnover". Journal of Applied Psychology, 62(2)

Mowday, R., Porter, L. \& Steers, R. (1982). Employee-Organization Linkages: The Psychology of Commitment, Absenteeism, and Turnover. Academic Press, New York. Recuperado de: https://bit.ly/3dF4WUT

Naupas, H. et. al. (2014). Metodología de la investigación Cuantitativa y Cualitativa y redacción de tesis. Ediciones de la Univ. Transversal. 4ta ed. Bogotá - Colombia.

ORGANIZACIÓN PARA LA COOPERACIÓN Y EL DESARROLLO ECONÓMICO (OCDE), MTSS (1987). Flexibilidad y mercado de trabajo: el debate actual. Colección de informes OCDE, 23.

Palomino, N. (2016). Consecuencias Jurídicas y Económicas que se Derivan de la Aplicación del Régimen Especial CAS al Personal de la Municipalidad Provincial de Trujillo 2016, [Tesis para optar el Grado de Maestra en Derecho, Trujillo]. 
Price, J., y Mueller, C. (1981). “A causal model of turnover for nurses”. Academy of Management Journal, 34(2)

Porter, L., y Steers, R. (1973). Organizational, Work and Personal Factors in Employee Turnover and Absenteeism. Irvine: [University of California].

Quecedo, R. y Castaño, C. (2002) Introducción a la metodología de investigación cualitativa. Recuperado de: https://bit.ly/2BOtUnj

Quintana, A. y Montgomery, W. (Eds.) (2006). Psicología: Tópicos de actualidad. Lima: UNMSM. Recuperado de: https://bit.ly/2BOFMpK.

Ramírez, M. (2012). Transformaciones del Estado en el gobierno local: La nueva gestión pública en Medellín. Reflexión Política, vol. 14, núm. 28, diciembre, 2012, Universidad Autónoma de Bucaramanga. Recuperado de: https://bit.ly/3ilfoOX

Ricoy, C. (2006). Contribución sobre los paradigmas de investigación Educativa. Revista do Centro de Educação, vol. 31, núm. 1, 2006, [Universidade Federal de Santa Maria], RS, Brasil. Recuperado de: https://bit.ly/388gb72

Robbins, P. y Judge, A. (2009) Organizational behavior. 13th Edition, México: Pearson Prentice Hall. Recuperado de: https://bit.ly/2ZCHNOp

Robbins, S. y Coulter, M. (2010). Administración. Décima edición. Ed. Prentice Hall. México DF. Recuperado de: https://bit.ly/2ZCHNOp

Robbins, S. y Coulter, M. (2013). Administración (12a. ed.). México. Pearson Educación.

Rodríguez, A. (1998) Introducción a la psicología del trabajo. Madrid. España.

Rodríguez, G., Gil, J. y García, E. (1996). Metodología de la investigación cualitativa, [Universidad de Cádiz -España].

Rodríguez, J. (2008). Análisis de los regímenes laborales en el sector público. Lima - Perú.

Rueda, S. (2011). La inconstitucionalidad del contrato administrativo de servicios - CAS. [Tesis doctoral. Universidad San Martin de Porres].

Ruiz, R. (2017). Desempeño laboral y modalidad contractual de los servidores del Ministerio Público - Gerencia Administrativa de San Martín, Moyobamba - 2017, [Universidad Cesar Vallejo. Tarapoto].

Sánchez, F. (2003). Constitución política, acuerdo nacional y planeamiento estratégico en el Perú. CEPAL - ILPES. Santiago de Chile. Recuperado de: https://bit.ly/2DlyWIR.

Sánchez, P. (2017). Cognifit Salud, Cerebro \& Neurociencia. Recuperado El 11 De Junio de 2017 de Https://Bit.ly/3lTedhu.

Servir (2017). Disponible en https://www.servir.gob.pe

Sonnentag, S., Volmert, J. \& Spychala, A. (2008). Job performance. C. L. Cooper y J. Barling (Eds.), The SAGE Handbook of Organizational Behavior (Vol 1: Micro approaches). Los Angeles: Sage Publications.

Sonnentag, S. \& Frese, M. (2012). Dynamic performance. En C. L. Cooper y J. Barling (Eds.) The SAGE Handbook of organizational Behavior (vol 1: Micro approaches). Los Ángeles: Sage Publications. Quinta Edición. México.

Taylor, F. (1999), La desvinculación en las organizaciones.

Tett, R., \& Meyer, J. (1993). “Job satisfaction, organizational commitment, turnover intention, and turnover: Path analyses based on meta-analytic findings". Personnel Psychology, $46(2)$

Testa, G. (1973). Organización y Dirección del personal. Bilbao: Ediciones Deusto. 
Tostes, M., y Villavicencio, A. (2012). Flexibilización del Derecho del Trabajo y sus implicancias sobre las relaciones laborales en el Perú. Revista Derecho PUCP $\mathrm{N}^{\circ}$ 68. Recuperado de: https://bit.ly/2DVLhnx

Vandenberg, R. \& Scarpello, V. (1990). The matching model: An examination of the processes underlying realistic job previews. Journal of Applied Psychology. Recuperado de: https://bit.ly/3e5le9T.

Vázquez, S. (2001). Compromiso organizacional y satisfacción laboral: predictores de la intención del empleado de dejar la organización. Trabajo de grado de Licenciatura no publicado. [Universidad Católica Andrés Bello. Caracas].

Villegas, F. (2012) Causas de la rotación de personal de receptores pagadores de la región I metropolitana de una institución financiera. [Universidad Landivar. Guatemala].

Wayne, R. (2010). Administración de recursos humanos. Décimo primera edición. PEARSON EDUCACIÓN, México, 2010ISBN: 978-607-32-0203-9 Área: Administración y economía.

Yin R. K. (1994). Case study research Design and Methods. U.S.A. Sage.

Zimmerman L., y Frank, H. (2006). "Antecedentes de la rotación voluntaria del personal". Investigación Administrativa, núm. 97 